Vol. 42 (1990) [33-39]

\title{
RADIAL GROWTH AND BOUNDEDNESS FOR BLOCH FUNCTIONS
}

\section{A. Bonilla and F. Perez Gonzalez}

Let $B$ be the Bloch space of all those functions $f$ holomorphic in the open unit disc $D$ of the complex plane satisfying $\sup _{|z|<1}\left(1-|z|^{2}\right)\left|f^{\prime}(z)\right|<\infty$. We establish sufficient conditions for the boundedness of functions $f$ belonging to $B$ satisfying a certain uniform radial boundedness condition, and, by introducing a wide class of subsets $E$ of $\partial D$, which we call negligible sets for boundedness, we show that if $f \in B$ and there is a constant $K>0$ such that $\limsup _{x \rightarrow e^{i \theta}}|f(z)| \leqslant K$ for $e^{i \theta} \in \partial D \backslash E$, then $f$ is bounded in D. Hence a significant extension of a theorem of Goolsby is obtained.

\section{INTRODUCTION}

As usual, $B$ denotes the Bloch space of all those holomorphic functions $f$ in the open unit disc $\mathbf{D}$ of the complex plane $C$ which satisfy

$$
\|f\|_{B}=|f(0)|+\sup _{|z|<1}\left(1-|z|^{2}\right)\left|f^{\prime}(z)\right|<\infty \text {. }
$$

Endowed with the Bloch norm $\|\cdot\|_{B}, B$ is a Banach space. The space $H^{\infty}(\mathbf{D})$ of all bounded analytic functions in $\mathbf{D}$ is strictly contained in $B$, since the function $\log (1+z) /(1-z) \in B \backslash H^{\infty}(\mathrm{D})$. T will denote the unit circle.

The following result was shown in [5]:

Theorem A. (Goolsby). Let $E$ be a finite subset of $T$, and let $f \in B$. If there exists a constant $K>0$ such that

$$
\limsup _{z \rightarrow a}|f(z)| \leqslant K
$$

for any $a \in \mathrm{T} \backslash E$, then $f$ is bounded in $\mathbf{D}$.

The proof of this result depends on Theorem 4.2 in [1] which we reproduce below.

Received 17th August 1989

We are grateful to Professor José L. Fernández who attracted our attention to Dahlberg's paper [3]. I. Marrero brought us the example after Theorem 1. We also give thanks to her. Partially supported by Consejería de Educación, Gobierno Autónomo de Canarias, proyecto núm. 08/30.04.86.

Copyright Clearance Centre, Inc. Serial-fee code: 0004-9729/90 \$A2.00+0.00. 
Theorem B. (Anderson, Clunie, Pommerenke). Let $f \in B$ and let $\Gamma$ be an arc ending at $e^{i \theta}$. Let $A \subseteq \mathrm{C}$. If

$$
\lim _{\substack{z \rightarrow e^{i \theta} \\ z \in \Gamma}} \operatorname{dist}[f(z), A]=0,
$$

then

$$
\limsup _{r \rightarrow 1-0} \operatorname{dist}\left[f\left(r e^{i \theta}\right), A\right] \leqslant K_{1}\|f\|_{B},
$$

where $K_{1}$ is an absolute constant.

The constant $K_{1}$ comes from the Lehto-Virtanen maximum principle (see [1], p.30 or [7]), and depends neither on the point $e^{i \theta}$ nor on the function $f$. In fact, if for a fixed $\beta>0$, we consider the expression (of Lehto-Virtanen):

$$
\delta_{0}(\alpha, \beta)=\frac{\sin \beta}{\beta}\left[1+\sqrt{1+\left(\frac{\alpha \beta}{\sin \beta}\right)^{2}}\right] \cdot \exp \left[-\sqrt{1+\left(\frac{\alpha \beta}{\sin \beta}\right)^{2}}\right]
$$

and choose some number $\alpha>0$ such that $\delta_{0}(\alpha, \beta) \geqslant 1$, we can assume $K_{1}=3 / \alpha$. In particular, for $\beta=3 \pi / 4$, we have $\delta_{0}(\alpha, \beta) \geqslant 1$ whenever $\alpha \leqslant 0.0001989$, and hence, if $\alpha=1 / 10$, then $K_{1}=30$. From now on $K_{1}$ will always represent an absolute constant.

Our major goal in this research has been to obtain a significant generalisation of Theorem A for subsets $E$ of $T$ bigger than a finite set. In Section 3 of this paper we show that this is the case (Theorem 3). In fact, Theorem 3 is derived from the results in Section 2, which is devoted to the establishing of sufficient conditions for the boundedness of functions $f \in B$ satisfying a certain radial uniform boundedness condition. To the best of our knowledge, although Theorem 2 is a corollary of [3], Theorem 4, the theorems in this section are new and cannot be deduced from any recent result in this area. (See $[4,6,8])$. Although Theorem 1 is a particular case of Theorem 2, we have included an independent proof based upon the ideas and geometric constructions in [1] and [5]. This allows us to extend Theorem A by using the same methods invoked in its proof.

\section{RADIAL GROWTH AND BOUNDEDNESS}

We shall need two lemmas.

LEMMA 1. ([5], p.720). Let $\left(r_{n}\right)$ be a sequence of real numbers in D such that $\lim _{n \rightarrow \infty} r_{n}=1$. Then there exists a sequence of discs, $\Delta_{n}$, satisfying:
(a) $r_{n} \in \Delta_{n}$;
(b) $1 \notin \bar{\Delta}_{n}$;
(c) the angle between $T$ and $\partial \triangle_{n}$ is $3 \pi / 4$;
(d) diameter $\left(\Delta_{n}\right) \longrightarrow 0$, as $n \longrightarrow \infty$; and,
(e) $\Delta_{n} \cap(C \backslash D) \neq 0$. 
REMARK. The construction of the $\triangle_{n}$ 's can be made in such a way that, for each $n$, the two points in $\partial \Delta_{n} \cap T$ remain in the semiplane $\{z: \operatorname{Im} z>0\}$.

LEMma 2. Let $f \in B$ and assume that there is a point $e^{i \theta_{0}}$ such that $\limsup _{r \rightarrow 1-0}\left|f\left(r e^{i \theta_{0}}\right)\right|<\infty$. Suppose also that $U$ is a domain in $C$ such that

(i) $e^{i \theta_{0}} \in \bar{U}$, and

(ii) $\bar{U}$ does not meet the segment $\left[r_{0}^{i \theta_{0}}, e^{i \theta_{0}}\right)$ for some $r_{0}, 0 \leqslant r_{0}<1$.

If $A=f(\bar{U} \backslash \mathrm{T})$, then

$$
\underset{r \rightarrow 1-0}{\limsup } \operatorname{dist}\left[f\left(r e^{i \theta_{0}}, A\right)\right] \leqslant K_{1}\|f\|_{B} .
$$

Proof: We shall exploit ideas from [1] and [5]. Without loss of generality, we suppose $\theta_{0}=0$, and $\|f\|_{B} \leqslant 1$. Further we assume that, at least, $U$ peaks at 1 across the domain $R=D \cap\{z: \operatorname{Im} z>0\}$. Let $\beta=3 \pi / 4$ and choose $\alpha$ so small that $\delta_{0}(\alpha, \beta) \geqslant 1$ is as in (1.1), and put $K_{1}=3 / \alpha$.

If (2.1) fails, then by the hypothesis on radial boundedness, a complex number $w_{0}$ and a sequence $\left(r_{n}\right)$ of real numbers with $r_{n} \rightarrow 1-0$ as $n \rightarrow \infty$, can be found such that

$$
\begin{gathered}
f\left(r_{n}\right) \longrightarrow w_{0}, \text { as } n \longrightarrow \infty \\
\operatorname{dist}\left[w_{0}, A\right]>K_{1} .
\end{gathered}
$$

Next, let $\left(\triangle_{n}\right)$ be a sequence of discs having the properties stated in Lemma 1 and the Remark above. Let $A_{n}$ and $C_{n}$ be the two arcs of $\partial \Delta_{n}$ in $R$. Since $U$ is connected and $\bar{U} \cap\left[r_{0}, 1\right)$ is empty, there is a number $N$ such that, for any $n \geqslant N$, we have

$$
\begin{aligned}
& r_{n} \in\left[r_{0}, 1\right), \\
& A_{n} \cap U \neq 0, \\
& C_{n} \cap U \neq 0 .
\end{aligned}
$$

If $G_{n}$ denotes the (open) connected component of $(C \backslash \bar{U}) \cap \triangle_{n}$ containing $r_{n}$, then $\bar{G}_{n} \subset \bar{\triangle}_{n}$ and $1 \notin \partial G_{n} \subset \partial \triangle_{n} \cup(\bar{U} \backslash 1)$. We assume for the moment the crucial inclusion

$$
\bar{G}_{n} \subset \mathbf{D} \text {. }
$$

Then $\partial G_{n} \subseteq \partial \triangle_{n} \cup(\bar{U} \backslash T)$, and if $g(z)$ is defined to be

$$
g(z)=\frac{1}{\alpha\left(f(z)-w_{0}\right)}, \quad z \in \mathrm{D},
$$


then $g$ is a meromorphic function which is normal:

$$
\left(1-|z|^{2}\right) \frac{\left|g^{\prime}(z)\right|}{1+|g(z)|^{2}}=\frac{\left(1-|z|^{2}\right) \alpha\left|f^{\prime}(z)\right|}{\alpha^{2}\left|f(z)-w_{0}\right|^{2}+1} \leqslant \alpha\left(1-|z|^{2}\right)\left|f^{\prime}(z)\right| \leqslant \alpha\|f\|_{B} \leqslant \alpha .
$$

Now, if $z \in \partial G_{n} \backslash \partial \triangle_{n}$, then $z \in \bar{U} \backslash T$, so that $f(z) \in A$, and the inequality in (2.2) leads to $|g(z)| \leqslant 1 /\left(\alpha K_{1}\right)=1 / 3$. We deduce from the Lehto-Virtanen maximum principle that $g$ is analytic and bounded in every $G_{n}$, with a bound independent of $n$. However $\left|g\left(r_{n}\right)\right| \rightarrow \infty$, as $n \rightarrow \infty$, and we reach a contradiction.

It only remains to show the inclusion in (2.4). The construction of the sequence $\Delta_{n}$ makes it clear that $\triangle_{n} \cap(C \backslash R) \subseteq \mathrm{D}$, so we need only check that $\bar{G}_{n} \cap R \subset \mathrm{D}$. If this were not the case, there would exist a point $q$ in $\bar{G}_{n} \cap R$ with $|q| \geqslant 1$. We may assume $q$ is on $\mathrm{T}$, for if $q$ were off $\mathrm{D}$ the arcwise connectedness of $G_{n}$ would yield an arc in $G_{n}$ joining $q$ with $r_{n}$, which would intersect $\mathrm{T}$. Next, let $E_{1}$ and $E_{2}$ be the components of $R \cap\left(C \backslash \triangle_{n}\right)$ whose closures contain $A_{n}$ and $C_{n}$ respectively. By (2.3), there are two points $t \neq 1$ and $s \neq 1$ such that $t \in E_{1} \cap U$ and $s \in E_{2} \cap U$. Since $U$ is connected, we can take a curve $\Gamma_{1}$ defined in $[0,1]$ such that $\Gamma_{1} \subseteq U, \Gamma_{1}(0)=t$ and $\Gamma_{1}(1)=s$. Let $x_{1}=\sup \left\{x \in[0,1] / \Gamma_{1}(x) \in A_{n}\right\}$ and let $x_{2}=\inf \left\{x \in\left[x_{1}, 1\right]: \Gamma_{1}(x) \in C_{n}\right\}$. Since $\Gamma_{1}(1) \notin A_{n}, x_{1} \neq 1$ and $\Gamma_{1}\left(x_{1}\right) \in A_{n}$. Similarly $\Gamma_{1}\left(x_{2}\right) \in C_{n}$, and $x_{1} \neq x_{2}$. Let $\Gamma$ be the closed (Jordan) curve formed by $\Gamma_{1}$ on $\left[x_{1}, x_{2}\right]$ and $\partial \triangle_{n}$ so that $r_{n}$ is in the interior of $\Gamma$. Since $\Gamma_{1} \subseteq U \subseteq D, q$ does not meet $\Gamma_{1 \mid\left[x_{1}, x_{2}\right]}$. This means that if $q^{\prime} \in D(q, r) \cap G_{n}$, for some $r>0$, then $q^{\prime}$ can be joined to $r_{n}$ by a curve $\gamma$ in $G_{n}$. But since $r_{n}$ is in the interior of $\Gamma$ and $q^{\prime}$ is outside $\Gamma$, we would have that $\Gamma_{1} \cap \gamma \neq \emptyset$, which is impossible because $\gamma \subseteq G_{n} \subseteq\left[\Delta_{n} \cap(C \backslash \bar{U})\right]$ and $\Gamma_{1} \subseteq U$.

We now establish our first theorem.

THEOREM 1. Let $f \in B$. If there is a constant $K>0$ such that

$$
\limsup _{r \rightarrow 1-0}\left|f\left(r e^{i \theta}\right)\right| \leqslant K
$$

for any $e^{i \theta}$ in $\mathrm{T}$, then $f$ is bounded in $\mathrm{D}$.

Proof: Suppose that $f$ is not bounded, and let $M$ be an arbitrary constant, $M>K_{1}\|f\|_{B}+K$. Since $\underset{r \rightarrow 1-0}{\limsup }\left|f\left(r e^{i \theta}\right)\right| \leqslant K$ for any $e^{i \theta}$, it follows that

$$
\underset{r \rightarrow 1-0}{\limsup } \operatorname{dist}\left[f\left(r e^{i \theta}\right), C \backslash \bar{D}(O, M)\right]>K_{1}\|f\|_{B}
$$

for each point $e^{i \theta}$. Let $U=f^{-1}(C \backslash \bar{D}(0, M))$. We shall assume that $U \neq \emptyset$, for otherwise $f$ would be bounded. 
Let $V$ be any component of $U$. If $\partial V \cap \mathrm{T}=\emptyset$, then $\bar{V} \subseteq \mathrm{D}$ and $f$ attains its maximum in $\bar{V}$ in a point $z_{0} \in(\partial V \backslash \mathrm{T}) \cap \mathrm{D}$, with $\left|f\left(z_{0}\right)\right|>M$; by continuity, $|f|>M$ in a disc $D\left(z_{0}, \varepsilon\right) \subseteq D$. This contradicts the maximality of $V$ and shows that $\partial V \cap \mathrm{T} \neq \emptyset$.

Next, let $e^{i \alpha}$ be any fixed point in $\partial V \cap T$. The condition on radial boundednes guarantees that there exists an $r_{0}, 0 \leqslant r_{0}<1$, such that $\bar{V} \cap\left[r_{0} e^{i \theta}, e^{i \alpha}\right)=\emptyset$. Put $A=f(\bar{V} \backslash \mathrm{T})$. By Lemma 2,

$$
\limsup _{r \rightarrow 1-0} \operatorname{dist}\left[f\left(r e^{i \alpha}, A\right)\right] \leqslant K_{1}\|f\|_{B} .
$$

Since $f(\bar{V} \backslash \mathrm{T}) \subseteq \mathrm{C} \backslash D(O, M)$, we find that $\underset{r \rightarrow 1-0}{\limsup \operatorname{dist}}\left[f\left(r e^{i \alpha}\right), \mathrm{C} \backslash \bar{D}(O, M)\right] \leqslant$ $K_{1}\|f\|_{B}$, contradicting (2.5).

We do not know whether Theorem 1 holds when the condition $f \in B$ is replaced by $f \in L_{a}^{1}$, the Bergman space of all those analytic functions in $D$ that are $L^{1}$ with respect to Lebesgue area measure. In this direction the following example shows that it is not true for an arbitrary analytic function in $D$.

Let $f$ be the function defined by

$$
f(z)=(1-z) \exp \left[-\exp \left(\frac{1+z}{1-z}\right)\right], \quad z \in \mathrm{D} .
$$

It is not hard to check that $f^{*}\left(e^{i \theta}\right)=\lim _{r \rightarrow 1-0} f\left(r e^{i \theta}\right)$ exists and $\left|f^{*}\left(e^{i \theta}\right)\right| \leqslant 2 e$, for any $e^{i \theta} \in \mathrm{T}$. Now let us consider the sequence $a_{k}=((k-1)+i \pi) /((k+1)+i \pi)$, $k=1,2, \ldots$ Then $a_{k} \in \mathrm{D}$ and $\left|f\left(a_{k}\right)\right|^{2}=4 /\left((1+k)^{2}+\pi^{2}\right) e^{2 e^{k}} \rightarrow \infty$, as $k \rightarrow \infty$. This implies that $f \notin H^{\infty}(D)$. Further, $f \notin L_{a}^{1}$ because the area integral of $|f|$ on discs centred at $a_{k}$ and with radii $\left(1-\left|a_{k}\right|\right) / 2$ can become very large as $k \rightarrow \infty$.

$\mathrm{O}_{\mathrm{n}}$ the other hand, we have the next result which is a consequence of [3], Theorem 4. As usual, "Dim" means Hausdorff dimension.

THEOREM 2. Let $f \in B$ and let $E$ be a subset of $\mathrm{T}$ with $\operatorname{Dim}(E)<1$. If there exists a constant $K>0$ such that $\limsup _{r \rightarrow 1-0}\left|f\left(r e^{i \theta}\right)\right| \leqslant K$ for any $e^{i \theta} \in T \backslash E$, then $f$ is bounded.

Proof: Since $f \in B,|f(z)| \leqslant C \log 1 /(1-|z|)$. Hence the function $u(z)=$ $\log |f(z)|$ satisfies $u(z) \leqslant \log \log 1 /(1-|z|)+C$. In particular, $u^{*}\left(e^{i \theta}\right)=$ $\limsup _{r \rightarrow 1-0} u\left(r e^{i \theta}\right) \leqslant$ constant for any $e^{i \theta} \in T \backslash E$, and $M(r, u)=\operatorname{Max}_{\theta} u\left(r e^{i \theta}\right)=$ $o\left(1 /\left((1-r)^{\alpha}\right)\right)$ for each $\alpha>0$. Choosing $\alpha_{0}$ such that $0<\alpha_{0}<1-\operatorname{Dim}(E)$, Theorem 4 in [3] shows that $u \leqslant$ constant in $D$. 


\section{NEGLIGIBLE SETS FOR BOUNDEDNESS}

In order to extend Theorem $A$ it will be convenient to introduce a class of subsets of $\mathbf{T}$.

Definition: Let $E$ be a subset of $\mathrm{T}$. We shall say that $E$ is a negligible set for boundedness (in short, negligible) if for every $e^{i \theta} \in E$, there is a subarc $I \subseteq T$ ending at $e^{i \theta}$ and $I \subseteq \mathrm{T} \backslash E$.

It is clear that any negligible set is a countable set, and that any subset of a negligible set is also negligible. We list some examples.

(i) Every finite set in $T$ is plainly negligible.

(ii) Let $E=\left(e^{i \theta_{n}}\right)$ be a sequence of points on $\mathrm{T}$ whose arguments converge strictly to $\theta_{0} \in[0,2 \pi]$. Then $E$ is negligible.

(iii) Let $E=\left(e^{i \theta_{n}}\right) \cup\{1\}$, where $2 \pi>\theta_{n} \rightarrow 0$ and $\theta_{n+1}-\theta_{n} \simeq 1 /\left(n(\log n)^{2}\right)$ for each $n$. Then $E$ is negligible but not a Carleson set.

We are in a position to prove the following result in two independent ways.

Theorem 3. Let $f \in B$ and let $E$ be a negligible set. If there is a constant $K>0$ such that

$$
\limsup _{z \rightarrow e^{i \theta}}|f(z)| \leqslant K
$$

for any $e^{i \theta} \in \mathrm{T} \backslash E$, then $f$ is bounded in $\mathrm{D}$.

Proof: First of all since $\operatorname{Dim}(E)=0$, the conclusion follows immediately from Theorem 2. On the other hand, we note that if $J$ is a subarc of $T \backslash E$ and $e^{i \theta_{0}}$ is an endpoint of $J$, then

$$
\limsup _{f \rightarrow 1-0}\left|f\left(r e^{i \theta_{0}}\right)\right| \leqslant K_{1}\|f\|_{B}+K+2
$$

this can be proved just as in [5], Theorem 2.60. But the same holds for any $e^{i \theta}$ belonging to $E$, since there exists an arc I ending at $e^{i \theta}$. If $M$ is a constant satisfying $M>$ $K_{1}\|f\|_{B}+K+2$, then $\limsup _{r \rightarrow 1-0}\left|f\left(r e^{i \alpha}\right)\right| \leqslant M$ for any $\alpha$. Now Theorem 1 implies that $f$ is bounded.

\section{REFERENCES}

[1] J.M. Anderson, J. Clunie and C. Pommerenke, 'On Bloch functions and normal functions', J. Reine. Angew. Math. 270 (1974), 12-37.

[2] J.A. Cima, 'The basic properties of Bloch functions', Internat. J. Math. Math. Sci. 2-3 (1979), 369-413. 
[3] B.E.I. Dahlberg, 'On the radial boundary values of subharmonic functions', Math. Scand. 40 (1977), 301-317.

[4] D. Girela, 'Integral means and radial growth of Bloch functions', Math. $Z .195$ (1987), 37-50.

[5] R.C. Goolsby, 'Boundedness for Bloch functions', Rocky Mountain J. Math. 16 (1986), 717-726.

[6] B. Korenblum, 'Estimates and radial growth of Bloch functions', Bull. Amer. Math. Soc. (N.S.) 12 (1985), 99-102.

[7] O. Lehto and K.I. Virtanen, 'Boundary behaviour and normal meromorphic functions', Acta Math. 87 (1957), 47-65.

[8] N.G. Makarov, 'On the distortion of boundary sets under conformal mappings', Proc. London Math. Soc. 51 (1985), 369-384.

Departamento de Análisis Matemático

Facultad de Matemáticas

Universidad de La Laguna

38271 La Laguna, Tenerife

Spain 